\title{
Inhibition of PI3K/Akt/mTOR overcomes cisplatin resistance in the triple negative breast cancer cell line HCC38
}

\author{
Katharina Gohr, Alexandra Hamacher, Laura H. Engelke and Matthias U. Kassack*
}

\begin{abstract}
Background: Widely established targeted therapies directed at triple negative breast cancer (TNBC) are missing. Classical chemotherapy remains the systemic treatment option. Cisplatin has been tested in TNBC but bears the disadvantage of resistance development. The purpose of this study was to identify resistance mechanisms in cisplatin-resistant TNBC cell lines and select targeted therapies based on these findings.

Methods: The TNBC cell lines HCC38 and MDA-MB231 were subjected to intermittent cisplatin treatment resulting in the 3.5-fold cisplatin-resistant subclone HCC38CisR and the 2.1-fold more resistant MDA-MB231CisR. Activation of pro-survival pathways was explored by immunostaining of phospho-receptor tyrosine kinases. Targeted therapies (NVP-AEW541, lapatinib and NVP-BEZ235) against activated pathways were investigated regarding cancer cell growth and cisplatin sensitivity.

Results: In HCC38CisR and MDA-MB231CisR, phosphorylation of epidermal growth factor receptor (EGFR) and insulin-like growth factor 1 receptor (IGF1R) was observed. In HCC38CisR, treatment with NVP-AEW541 increased potency of lapatinib almost seven-fold, but both compounds could not restore cisplatin sensitivity. However, the dual phosphoinositide 3-kinase (PI3K) and mammalian target of rapamycin (mTOR) inhibitor NVP-BEZ235 acted synergistically with cisplatin in HCC38CisR and fully restored cisplatin sensitivity. Similarly, NVP-BEZ235 increased cisplatin potency in MDA-MB231CisR. Furthermore, NVP-AEW541 in combination with lapatinib restored cisplatin sensitivity in MDA-MB231CisR.

Conclusion: Simultaneous inhibition of EGFR and IGF1R in cisplatin-resistant TNBC cell lines was synergistic regarding inhibition of proliferation and induction of apoptosis. Co-treatment with NVP-BEZ235 or with a combination of NVP-AEW541 and lapatinib restored cisplatin sensitivity and may constitute a targeted treatment option for cisplatin-resistant TNBC.
\end{abstract}

Keywords: Triple negative breast cancer, HCC38, MDA-MB231, EGFR, IGF1R, NVP-AEW541, NVP-BEZ235, Lapatinib, Cisplatin resistance

\section{Background}

Breast cancer is the second most common cancer in the world and the incidence of female breast cancer has continuously increased [1]. In 2013, 1.8 million incident cases of breast cancer occurred, and the disease caused 464,000 deaths [1]. Triple negative breast cancer (TNBC) accounts for $10-20 \%$ of these breast cancer cases [2]. This type of breast cancer is defined by lacking protein expression of progesterone (PR) and estrogen receptors (ER) as well as by low ErbB2 expression. For

\footnotetext{
* Correspondence: Matthias.Kassack@uni-duesseldorf.de Institute for Pharmaceutical and Medicinal Chemistry, Heinrich Heine University Düsseldorf, Universitätsstraße 1, 40225 Düsseldorf, Germany
}

this reason, TNBCs cannot benefit from endocrine therapies or trastuzumab [3]. Therefore, chemotherapy is the systemic treatment option. The use of cisplatin and carboplatin in treatment of TNBCs is currently investigated in clinical trials and initial results indicate a beneficial effect for cisplatin in neoadjuvant chemotherapy $[4,5]$. One major challenge in cisplatin therapy is drug resistance which can be intrinsic or occur after several cycles of therapy. Trigger for cisplatin resistance can be found pre-target (e.g. reduced uptake), on-target (e.g. increased DNA-repair), post-target (e.g. inactivation of TP53) or off-target [6]. Off-target mechanisms include activation 
of pro-survival pathways mediated for example via growth factor receptors.

We have previously shown that resveratrol or ellagic acid prevented the development of cisplatin resistance in the ovarian cancer cell line A2780. This effect is at least in part based on the prevention of activation of ErbB2 and ErbB3 in the course of long-term cisplatin treatment [7]. IGF1R activation has also been shown to be a crucial step in the development of cisplatin resistance [8]. Activation of growth factor receptors may also play a role in the development of cisplatin resistance in TNBC and due to their involvement in cell proliferation, apoptosis and metastasis they are considered attractive targets for therapies beyond classical chemotherapeutic drugs [9]. In 1998, a link between elevated insulin-like growth factor 1 (IGF1) blood levels and breast cancer risk in premenopausal women has been published [10]. In this context the IGF1R emerged as a promising target in cancer therapy. Binding of its ligands to IGF1R results in the activation of mainly two downstream signaling networks: PI3K-Akt-mTOR and RAF-MAPK, both linked to cell survival and inhibition of apoptosis. Interestingly, not high expression but high phosphorylation of IGF1R was predictive for poor prognosis in breast cancer [11]. Extensive research in this area was done but after initially promising results, phase III clinical trials using anti-IGF1R-targeted therapies were mainly disappointing [12]. These findings might be due to resistance mechanisms like compensatory signaling via growth hormone receptors, insulin receptors or epidermal growth factor receptors. Therefore, combination therapies were suggested. In vitro studies showed a synergistic effect of a small molecule IGF1R inhibitor with gefitinib as EGFR/ ErbB2 inhibitor [13]. However, as has been seen for IGF1R inhibitors alone, larger clinical trials combining IGF1R inhibitors with either gefitinib or erlotinib failed [14]. Taking into account that no biomarkers were used to predict response, predictive tools for the use of IGF1R inhibitors might be necessary.

The purpose of our study was to identify resistance mechanisms in a cisplatin-resistant TNBC cell line leading to targeted therapies as treatment options in this cancer type. Evaluation of the phosphorylation status of receptor tyrosine kinases revealed activation of IGF1R and EGFR as a result of cisplatin resistance. Therefore, inhibitors of these two receptors (NVP-AEW541 and lapatinib) and an inhibitor of downstream acting PI3K/ Akt/mTOR (NVP-BEZ235) were evaluated regarding their effects on cancer cell growth and cisplatin sensitivity. Indeed, co-treatment of NVP-AEW541 with lapatinib increased potency of lapatinib in the cisplatinresistant TNBC cell line HCC38CisR but did not increase cisplatin sensitivity. On the other hand, NVPBEZ235 acted synergistically with cisplatin and fully restored cisplatin sensitivity in HCC38CisR. Furthermore, in the highly cisplatin-resistant TNBC cell line MDA-MB231CisR, treatment with NVP-BEZ235 or cotreatment of NVP-AEW541 with lapatinib increased potency of cisplatin up to 4.8 -fold.

\section{Methods \\ Materials}

NVP-AEW541 and NVP-BEZ235 were gifts from Novartis (Basel, Switzerland). Lapatinib, KU0063794 and LY294002 were from Cayman Chemical (Michigan, USA). Cisplatin was purchased from Sigma-Aldrich (Steinheim, Germany). 3-(4,5-Dimethylthiazol-2-yl)-2,5diphenyltetrazolium bromide (MTT) was purchased from Serva (Heidelberg, Germany). Propidium iodide was from PromoCell (Heidelberg, Germany). Roswell Park Memorial Institute (RPMI) media 1640, Dulbecco's Modified Eagle Medium (DMEM), fetal bovine serum (FBS), penicillin/streptomycin $[10,000 \mathrm{U} / \mathrm{ml} ; 10 \mathrm{mg} / \mathrm{ml}]$ and trypsin-EDTA (0.05\% trypsin, $0.02 \%$ EDTA in PBS) were purchased from PAN Biotech (Aidenbach, Germany). Primary antibodies were purchased from R\&D Systems (Wiesbaden, Germany) (pIGF1R, IGF1R, p-EGFR, EGFR, p-ErbB2, ErbB2, p-ErbB3, ErbB3) or Santa Cruz Biotechnology (Heidelberg, Germany) (p-Akt, Akt, $\beta$-Actin, PARP). HRP-conjugated secondary antibodies were from $R \& D$ Systems. All other reagents and chemicals were from VWR BDH PROLABO (Darmstadt, Germany).

\section{Cell lines and cell culture}

The triple negative breast cancer cell line HCC38 was obtained from ATCC (Manassas, USA, ATCC order number: ATCC ${ }^{\circ} \mathrm{CRL}^{2} 2314^{\mathrm{nx}}$ ) and cultivated in RPMI1640 medium supplemented with $10 \%$ FBS, $120 \mu \mathrm{g} / \mathrm{ml}$ streptomycin and $120 \mathrm{U} / \mathrm{ml}$ penicillin. The TNBC cell line MDA-MB231 (ATCC, Manassas, USA, ATCC order number: ATCC $^{\oplus}$ HTB- $^{\mathrm{mm}}{ }^{\mathrm{m}}$ ) was cultivated in DMEM supplemented with $15 \% \mathrm{FBS}, 120 \mu \mathrm{g} / \mathrm{ml}$ streptomycin and $120 \mathrm{U} / \mathrm{ml}$ penicillin. Cells were grown at $37{ }^{\circ} \mathrm{C}$ in a humidified atmosphere containing $5 \% \mathrm{CO}_{2}$. $\mathrm{HCC} 38 \mathrm{CisR}$ and MDA-MB231CisR, the cisplatin resistant subclones of HCC38 and MDA-MB231, respectively, were generated by intermittent treatment of HCC38 or MDAMB231 with cisplatin for 40 cycles according to methods previously published $[7,8,15]$. Cells were grown to $80-90 \%$ confluency before using them for assays.

\section{MTT cell viability assay}

Cell viability was determined using the MTT assay as previously described [7]. Resistance factor was calculated as ratio of $\mathrm{IC}_{50}$ of the resistant cell line and $\mathrm{IC}_{50}$ of the sensitive cell line. To investigate the effect of the small molecule inhibitors on cisplatin cytotoxicity, compounds 
were added $48 \mathrm{~h}$ prior to $72 \mathrm{~h}$ cisplatin treatment. For combination index analysis, cell viability was determined from each well relative to the average absorbance of control wells. The combination indexes (CIs) were calculated using CalcuSyn 2.1 software (Biosoft, Cambridge, U.K.) based on the Chou - Talalay method [16]. CI > 1 indicates antagonism. $\mathrm{CI}=1$ indicates an additive effect and $\mathrm{CI}<0.9$ indicates synergism.

\section{Neutral red cell viability assay}

To exclude compound effects potentially influencing mitochondrial activity, neutral red cell viability assay instead of MTT assay was performed as previously described [17]. Briefly, after incubation time, medium was removed and $200 \mu \mathrm{l}$ neutral red incubation solution (medium containing FBS, 0.1 M HEPES buffer $\mathrm{pH} 7.4$ and $0.01 \%$ neutral red) was added. After $2 \mathrm{~h}$, incubation solution was removed and cells were quickly washed with $1 \% \mathrm{CaCl}_{2} \times 2 \mathrm{H}_{2} \mathrm{O}$ in $1 \%$ formaldehyde solution. After a second washing step, cells were lysed with a 1:1 mixture of ethanol and 1\% acetic acid. Absorbance was measured at 544 and $690 \mathrm{~nm}$ in a FLUOstar microplate reader (BMG Labtech, Ortenberg, Germany).

\section{Doubling time}

The assay was performed as previously described [7]. Cells were seeded in 6-well plates (Sarstedt AG, Nürmbrecht, Germany). After 24, 48, 72, and 96 h, cells were trypsinized and washed with PBS. Total number of cells in $1 \mathrm{ml}$ buffer was counted in a CyFlow ${ }^{\circ}$ space (Partec, Muenster, Germany). Doubling time was calculated using GraphPad Prism (version 4, GraphPad Software Inc., San Diego, USA).

\section{Western blotting}

For western blotting, standard procedures were used as previously described [7].

\section{RTK signal pathway analysis}

The tyrosine-kinase phospho-proteom was investigated by a human phospho-receptor tyrosine kinase antibody array (Cat\# ARY001) from R\&D Systems according to the manufacturer's protocol. Cell lysate containing $300 \mu \mathrm{g}$ protein was used.

\section{Cell cycle analysis}

Distribution of cell cycle phases of the different cell lines was analyzed by flow cytometry using standard procedures as previously described [7].

\section{Apoptosis analysis}

Apoptotic cells were determined by propidium iodide staining as previously described [7].

\section{Scratch assay}

Scratch assay was performed according to standard procedures as previously described [7]. Cell-free area was determined using ImageJ [18]. Percentage of space that was occupied with cells after 24 h was calculated.

\section{Statistical analysis}

Assays were performed at least in three independent experiments. Concentration effect curves were then generated by nonlinear regression curve fitting using the 4-parameter logistic equation with variable hill slope (GraphPad Prism version 4, GraphPad Software Inc.). Data presented are mean \pm SEM if not otherwise stated. Statistical significance was assessed by two-tailed Student's t-test or ANOVA and considered significant if $p<0.05$. $\mathrm{pIC}_{50} \pm \mathrm{SEM}$ leading to the reported $\mathrm{IC}_{50}$ values are shown in Additional file 1.

\section{Results}

The cisplatin resistant cell line HCC38CisR was generated by weekly exposure to the $\mathrm{IC}_{50}$ of cisplatin for $6 \mathrm{~h}$. After 40 cycles, the $\mathrm{IC}_{50}$ (determined by MTT) has shifted from $2.7 \mu \mathrm{M}$ to $9.4 \mu \mathrm{M}$ corresponding to a resistance factor of 3.5 (Fig. 1a). This resistance factor is in the range of previously reported resistance factors of cell lines established from cancer patients before and after chemotherapy [19]. Resistance could be maintained without further cisplatin treatment. $\mathrm{IC}_{50}$ of cisplatin varied throughout the duration of these studies between 7 and $12 \mu \mathrm{M}$ for HCC38CisR. HCC38CisR was characterized in comparison to the parental cell line HCC38. Phospho-receptor tyrosine kinase antibody array was used to determine receptor activation. Other receptors than those shown in Fig. 1b (EGFR-family, IGF1R) were not differentially phosphorylated. HCC38 showed - as expected - no activation of ErbB2 but activation of EGFR and ErbB3. Cisplatin resistance (HCC38CisR) did not generate ErbB2 activation, while EGFR and IGF1R showed a markedly enhanced activation in HCC38CisR (Fig. 1b). In contrast, ErbB3 activation was diminished in HCC38CisR. These results could be confirmed by western blotting (Fig. 1c and d). In this assay, expression and activation of receptor tyrosine kinases (RTKs) was estimated in HCC38, HCC38CisR (long-term cisplatin stress, $40 \times$ intermittent $6 \mathrm{~h}$ cisplatin treatment), and HCC38 exposed to short-term cisplatin stress (6 h $\mathrm{IC}_{50}$ of cisplatin with $24 \mathrm{~h}$ or 1 week recovery). IGF1R and EGFR phosphorylation was increased after $6 \mathrm{~h}$ cisplatin stress and $24 \mathrm{~h}$ recovery, and in HCC 38CisR. If HCC38 treated $6 \mathrm{~h}$ with cisplatin could recover from cisplatin stress for one week, receptor phosphorylation decreased nearly to the initial state. Evaluating the expression of growth factor receptors, there was hardly any difference between untreated HCC38 and HCC38CisR. Akt 


\section{a}

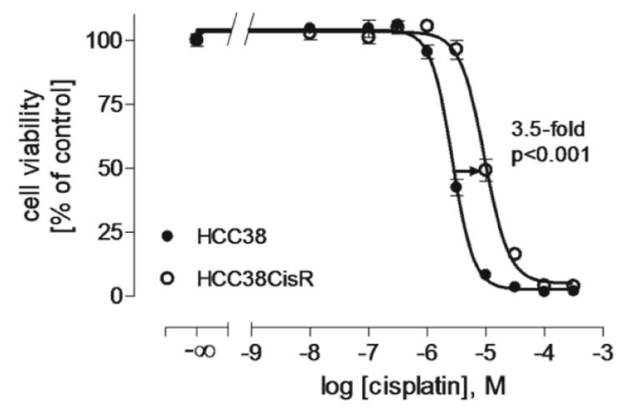

C

p-IGF1R (130 kDa)

EGFR (180 kDa)

p-EGFR (180 kDa)

ErbB3 (185 kDa)

p-ErbB3 (185 kDa)

Akt (62 kDa)

p-Akt (62 kDa)

$\beta$-Actin (42 kDa)

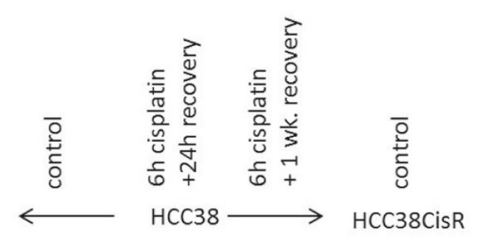

d
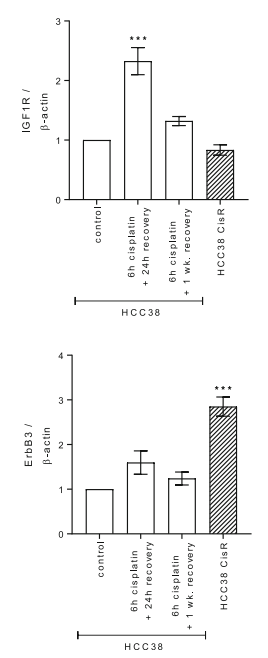

Fig. 1 (See legend on next page.) b

HCC38 HCC38CisR

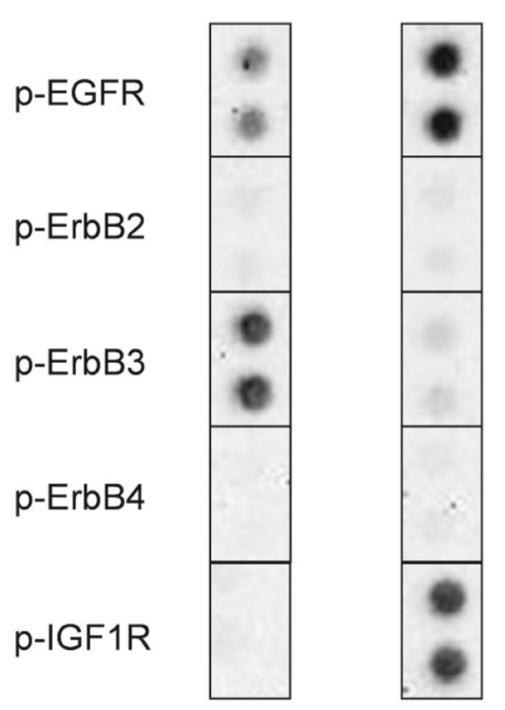

e
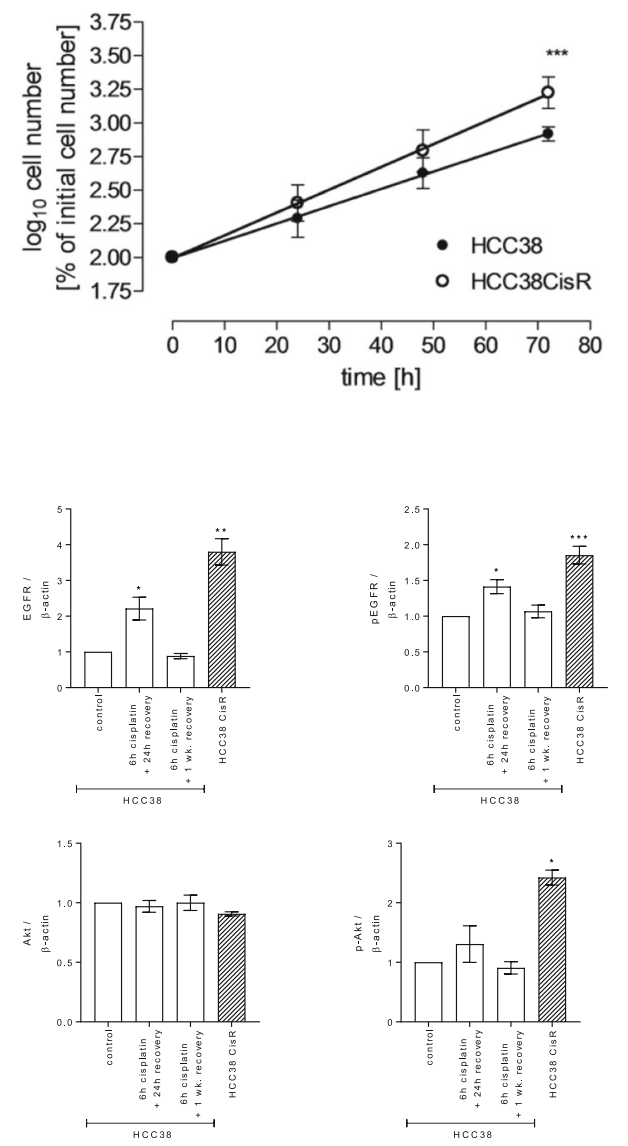


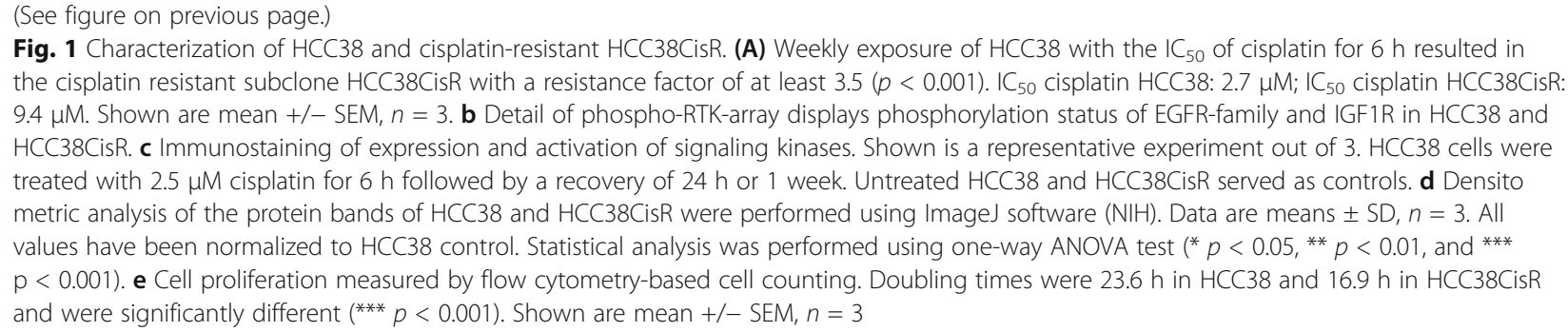

expression and phosphorylation was enhanced in HCC38CisR compared to HCC38 either untreated or short-term treated with cisplatin. Long-term cisplatin treatment resulting in HCC38CisR further increased proliferation rate and decreased doubling time significantly from $24 \mathrm{~h}$ to $17 \mathrm{~h}$ as displayed in Fig. 1e.

Based on activation of EGFR and IGF1R in HCC38CisR (Fig. 1), the dual EGFR/ErbB2 inhibitor lapatinib and the IGF1R inhibitor NVP-AEW541 were chosen for further experiments. The $\mathrm{IC}_{50}$ of both inhibitors was lower in HCC38CisR than in HCC38 (Fig. 2a/ b). The effect was more pronounced for NVP-AEW541 $(5.7 \mu \mathrm{M}$ vs. $2.3 \mu \mathrm{M})$ than for lapatinib $(9.2 \mu \mathrm{M}$ vs. $6.0 \mu \mathrm{M})($ Fig. 2a/b). Next, we tested the combination of both inhibitors. In HCC38, co-incubation of NVPAEW541 had no effect on the $\mathrm{IC}_{50}$ of lapatinib, and vice versa, coincubation of lapatinib had no effect on the $\mathrm{IC}_{50}$ of NVP-AEW541 (Fig. 2a/b). However, coincubation of NVP-AEW541 caused a significant increase in potency of lapatinib in HCC38CisR (almost 7-fold from 6.0 to $0.88 \mu \mathrm{M}$, Fig. 2a). Vice-versa, coincubation of lapatinib resulted in a significantly decreased $\mathrm{IC}_{50}$ for NVP-AEW541 in HCC38CisR (2-fold from 2.3 to $1.1 \mu \mathrm{M}$, Fig. 2b). To confirm the observed effects, synergism studies were performed (Table 1). Analysis based on the Chou-Talalay method [16] suggested a synergistic interaction between lapatinib and NVP-AEW541 (combination indexes $\mathrm{CI}<0.9$ ) in HCC38CisR.

Since MTT assay cannot distinguish between inhibition of proliferation and induction of apoptosis, we examined induction of apoptosis using propidium iodide nuclear staining (Fig. 2c). Both inhibitors were added alone or in combination for $48 \mathrm{~h}$ in a concentration of $2 \mu \mathrm{M}$. In HCC38 the treatment induced nearly no apoptotic cells (Fig. 2c). In HCC38CisR, NVP-AEW541 $(1.53 \pm 1.42 \%)$ and lapatinib $(2.59 \pm 0.83 \%)$ showed similarly nearly no induction of apoptosis whereas the combination of both compounds could heavily induce apoptosis $(28.7 \pm 2.62 \%$, Fig. 2c). The effect of this combination on cell cycle distribution in HCC38CisR was then determined using propidium iodide staining (Fig. 2d). Again, NVP-AEW541 and lapatinib alone or in combination were added in a concentration of $2 \mu \mathrm{M}$ for $48 \mathrm{~h}$ prior to ethanol fixation. NVP-AEW541 and lapatinib alone had no significant effects. In contrast, the combination of both compounds could reduce the fraction of cells in the $\mathrm{G}_{2} / \mathrm{M}$ phase from $25.7 \%$ to $14.2 \%$ while increasing the fraction of cells in $G_{1}$ phase from $62.8 \%$ to $77.7 \%$ ( $p<0.001$; Fig. 2 d). Treatment with lapatinib, NVP-AEW541 or their combination had no effect on cell cycle distribution in HCC38 (see Additional file 2). Next, the effect on phosphorylation of Akt, EGFR and IGF1R after $6 \mathrm{~h}$ treatment of HCC38CisR with an $\mathrm{IC}_{50}$ of lapatinib or NVP-AEW541 alone or in combination was determined by western blotting (Fig. 2e, f). Whereas both compounds alone had only moderate effects on receptor phosphorylation, their combination reduced EGFR and IGF1R phosphorylation to a greater extent. Interestingly, Akt phosphorylation was unaffected by either treatment.

Since EGFR and IGF1R were activated in cisplatinresistant $\mathrm{HCC} 38 \mathrm{CisR}$, we examined if the combination of lapatinib and NVP-AEW541 could restore cisplatin sensitivity in HCC38CisR (Fig. 2g). HCC38CisR was pretreated with the inhibitors $48 \mathrm{~h}$ prior to cisplatin treatment. The inhibitors alone and in combination had no significant effect on cisplatin sensitivity. In HCC38, the same was observed: neither lapatinib nor NVP-AEW541 alone nor their combination had an effect on cisplatin sensitivity (see Additional file 3).

It has been shown that cancer cells can easily switch membrane-bound RTK pathways upon inhibition of a particular RTK and still use the same downstream signaling pathways [20]. Further, since neither lapatinib nor NVP-AEW541 had an effect on cisplatin sensitivity and both compounds did not alter Akt phosphorylation increased in HCC38CisR (Fig. 2e, f), we tested whether NVP-BEZ235, a dual inhibitor of PI3K and mTOR, had an effect on cisplatin sensitivity. Evaluating the cytotoxicity of NVP-BEZ235 in HCC38 and HCC38CisR revealed that the $\mathrm{IC}_{50}$ was lower in $\mathrm{HCC} 38(9.1 \mathrm{nM})$ than in HCC38CisR (69.3 nM) (See Additional file 4). $48 \mathrm{~h}$ pretreatment with $20 \mathrm{nM}$ NVP-BEZ235 increased potency of cisplatin in HCC38CisR by a factor of 4 into the range of the non-resistant cell line $\mathrm{HCC} 38\left(\mathrm{IC}_{50}\right.$ HCC38CisR: $7.9 \mu \mathrm{M}$; $\mathrm{IC}_{50}$ HCC38CisR pretreated with 20 nM NVP-BEZ235: $2.0 \mu \mathrm{M}$; Fig. 3a). In HCC38, 20 nM NVP-BEZ235 had no effect on cisplatin 
a
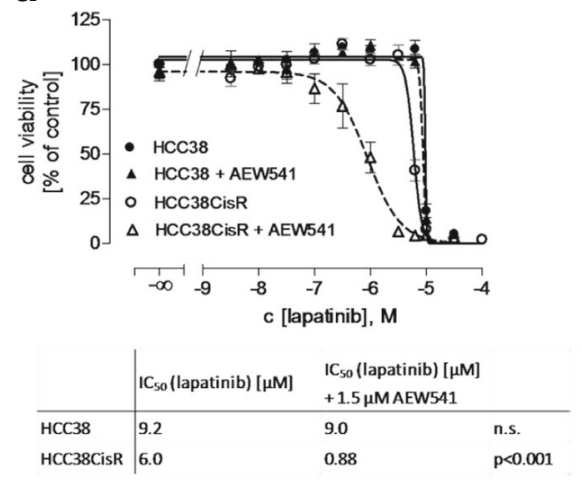

C

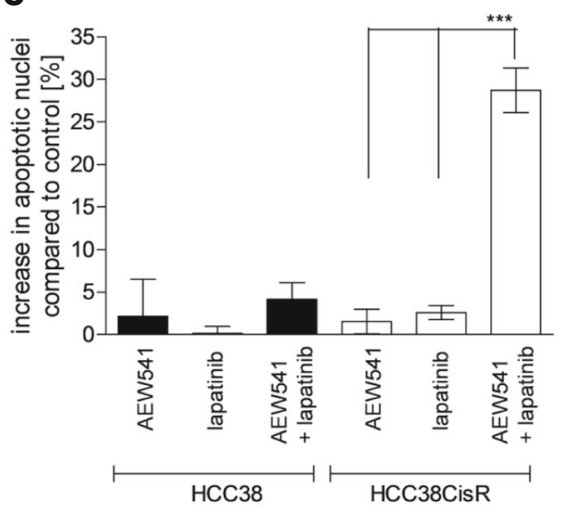

e

AEW541

p-EGFR (180 kDa)

p-IGF1R (130 kDa)

p-Akt (62 kDa)

$\beta$-Actin (42 kDa)

\section{f}
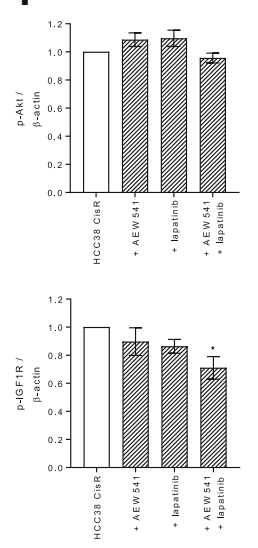

b
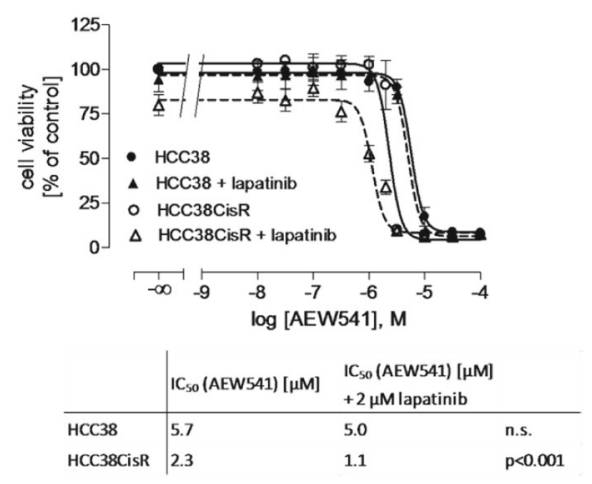

d

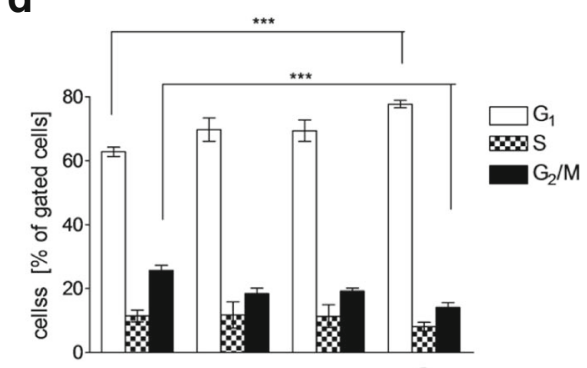

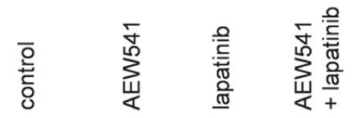

g

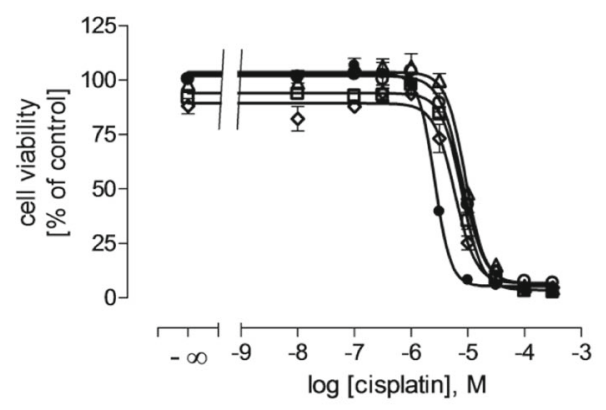

- $\mathrm{HCC} 38$

$\mathrm{IC}_{50} 2.6 \mu \mathrm{M}$

- HCC38CisR

$\mathrm{IC}_{50} 7.9 \mu \mathrm{M}$

$\triangle$ HCC38CisR + lapatinib $\quad I_{50} 9.1 \mu \mathrm{M}$

a HCC38CisR + AEW541 $\quad \mathrm{IC}_{50} 7.8 \mu \mathrm{M}$

- HCC38CisR + AEW541 + lapatinib $\mathrm{IC}_{50} 5.9 \mu \mathrm{M}$

Fig. 2 (See legend on next page.) 
(See figure on previous page.)

Fig. 2 Combination of lapatinib and NVP-AEW541 is hyper-additive but not reversing cisplatin resistance in HCC38CisR. a Coincubation with $1.5 \mu \mathrm{M}$ NVP-AEW541 significantly decreased IC 50 of lapatinib in HCC38CisR, whereas this treatment had no effect in HCC38. b Coincubation with $2 \mu \mathrm{M}$ lapatinib significantly decreased IC 50 of NVP-AEW541 in HCC38CisR but had no effect in HCC38. c In HCC38CisR (but not in HCC38), the combination of NVP-AEW541 and lapatinib significantly induced apoptosis in a hyper-additive manner $\left.{ }^{* * *} p<0.001\right)$. NVP-AEW541 and lapatinib were used at $2 \mu \mathrm{M}$. Cells were treated for $48 \mathrm{~h}$ and the amount of apoptotic nuclei in the control was subtracted from treated samples. $\mathbf{d}$ Effect of NVP-AEW541 or lapatinib ( $2 \mu \mathrm{M}$, respectively) on cell cycle in HCC38CisR. Combination of $2 \mu \mathrm{M}$ NVP-AEW541 and $2 \mu \mathrm{M}$ lapatinib significantly $\left({ }^{* * *} p<0.001\right)$ increased cell population in $G_{1}(77.7 \pm 1.2 \%$ vs. $67.3 \pm 1.4 \%)$ while reducing cell population in $G_{2} / M$ phase $(14.2 \pm 1.5 \%$ vs. $25.7 \pm 1.6 \%$ ). Incubation time was $48 \mathrm{~h}$. e Western blot analysis of p-EGFR, p-IGF1R, and p-Akt upon treatment of HCC38CisR with an IC $5_{50}$ of lapatinib or NVP-AEW541 or both compounds for $6 \mathrm{~h}$. $\mathbf{f}$ Densitometric analysis of the protein bands for p-AKT, p-EGFR, and p-IGF1R of HCC38CisR were performed using ImageJ software $(\mathrm{NIH})$. Data are means $\pm \mathrm{SD}, n=3$. All values have been normalized to untreated HCC38 CisR. Statistical analysis was performed using one-way ANOVA test (* $p<0.05$ ). $\mathbf{g}$ Effect of $1 \mu \mathrm{M}$ lapatinib and $1.5 \mu \mathrm{M}$ NVP-AEW541 on cisplatin sensitivity either alone or in combination. Lapatinib and/or NVP-AEW541 were added $48 \mathrm{~h}$ prior to cisplatin treatment. $\mathrm{I}_{50}$ of cisplatin did not significantly differ. All data shown are mean + /- SEM, $n=3$, except (e) showing a representative experiment out of 3

sensitivity (Fig. 3a). However, NVP-BEZ235 had a more pronounced effect on cell viability in $\mathrm{HCC} 38$ as observed by a reduction of the top plateau of the concentration effect curve to $48 \%$ in HCC38 versus $74 \%$ in HCC38CisR (Fig. 3a). To corroborate the observed effect in HCC38CisR, synergism studies were performed. The calculated CIs indicated synergism between cisplatin and NVP-BEZ235 in HCC38CisR (Table 2). Because NVPBEZ235 inhibits PI3K as well as mTOR, we examined the effect on cisplatin sensitivity of compounds inhibiting only one of these targets: LY294002 was chosen as PI3K inhibitor, KU0063794 as mTOR inhibitor (Fig. 3b). $48 \mathrm{~h}$ preincubation with either compound prior to cisplatin treatment could significantly $(p<0.001)$ sensitize HCC38CisR for cisplatin treatment by a factor of approximately 2. If both inhibitors LY294002 and KU0063794 were combined in $48 \mathrm{~h}$ preincubation prior to cisplatin treatment in $\mathrm{HCC} 38 \mathrm{CisR}$, the cisplatin $\mathrm{IC}_{50}$ of the parental cell line HCC38 was nearly restored (2.9 $\mu \mathrm{M}$, Fig. 3b). The effect of NVP-BEZ235 on cisplatin sensitivity was slightly, but significantly $(p<0.05)$ stronger than the effect of the combination of KU0063794 and LY294002.

Synergism between NVP-BEZ235 and cisplatin was observed in MTT (Table 2) and further verified by western blotting (Fig. 3c) and apoptosis assay (Fig. 3d). $48 \mathrm{~h}$ preincubation with $20 \mathrm{nM}$ NVP-BEZ235 followed by a $6 \mathrm{~h}$ treatment with $3 \mu \mathrm{M}$ cisplatin led to a markedly enhanced accumulation of cleaved poly ADP-ribose

Table 1 Synergism studies between NVP-AEW541 and lapatinib

\begin{tabular}{llllll}
\hline Lapatinib $[\mu \mathrm{M}]$ & & & & & \\
\hline AEW541 $[\mu \mathrm{M}]$ & 1 & 2 & 2.5 & 3 & 3.5 \\
\hline 0.5 & $\mathrm{a}$ & $\mathrm{a}$ & 0.76 & 0.52 & 0.46 \\
1 & $\mathrm{a}$ & 0.58 & 0.51 & 0.44 & 0.42 \\
1.5 & 0.96 & 0.59 & 0.58 & 0.51 & 0.49 \\
2 & 0.46 & 0.42 & 0.46 & 0.49 & 0.52 \\
3 & 0.69 & 0.57 & 0.56 & 0.68 & 0.64 \\
\hline${ }^{a}$ fraction affected $<0.2$ & & & & &
\end{tabular}

polymerase (PARP) in HCC38CisR serving as an indicator of caspase 3 activation. Whereas either compound alone could not induce PARP cleavage, the combination of NVP-BEZ235 and cisplatin markedly induced PARP cleavage. This effect was not observed in HCC38 (see Additional file 5). Similarly NVP-BEZ235 could enhance the number of cisplatin-induced apoptotic nuclei significantly (hyper-additive) without having an own pronounced apoptotic effect. Whereas cisplatin alone caused $11.4 \%$ apoptotic nuclei, addition of NVP-BEZ235 tripled this effect (35.3\%). Again, this effect could not be observed in HCC38 (see Additional file 6).

Since the effect of NVP-BEZ235 on its different targets is concentration-dependent [21], we tested a low $(20 \mathrm{nM})$ and a high $(280 \mathrm{nM})$ concentration of NVPBEZ235 on EGFR, IGF1R and Akt phosphorylation (Fig. 3e, f) in HCC38CisR. $280 \mathrm{nM}$ NVP-BEZ235 reduced Akt phosphorylation whereas $20 \mathrm{nM}$ had no effect. Further, phosphorylation of IGF1R and EGFR was diminished, particularly at $280 \mathrm{nM}$ NVP-BEZ235. Cell cycle was only affected by $280 \mathrm{nM}$ (but not $20 \mathrm{nM}$ ) NVPBEZ235 in HCC38CisR (Fig. 3g): cells in $G_{2} / M$ phase slightly increased compared to control $(28.3 \%$ versus $23.6 \%$ accompanied by a slight decrease of cells in $\mathrm{G}_{1}$ phase $(60.0 \%$ versus $67.3 \% ; p<0.05$; Fig. $3 \mathrm{~g})$.

Eventually, we studied effects of the examined kinase inhibitors NVP-AEW541, lapatinib and NVP-BEZ235 on the migratory potential of HCC38CisR by a scratch assay (Fig. 4). $24 \mathrm{~h}$ after applying a scratch to untreated cells,

Table 2 Synergism studies between cisplatin and NVP-BEZ235

\begin{tabular}{llllll}
\hline BEZ235 [nM] & & & & & \\
\hline cisplatin $[\mu \mathrm{M}]$ & 30 & 40 & 50 & 60 & 70 \\
\hline 1 & $a$ & $a$ & 0.74 & 0.69 & 0.76 \\
2 & 0.76 & 0.70 & 0.71 & 0.60 & 0.66 \\
3 & 0.76 & 0.59 & 0.61 & 0.60 & 0.64 \\
5 & 0.71 & 0.63 & 0.62 & 0.61 & 0.67 \\
7 & 0.70 & 0.59 & 0.61 & 0.60 & 0.67 \\
\hline afraction affected $<0.2$ & & & &
\end{tabular}


a

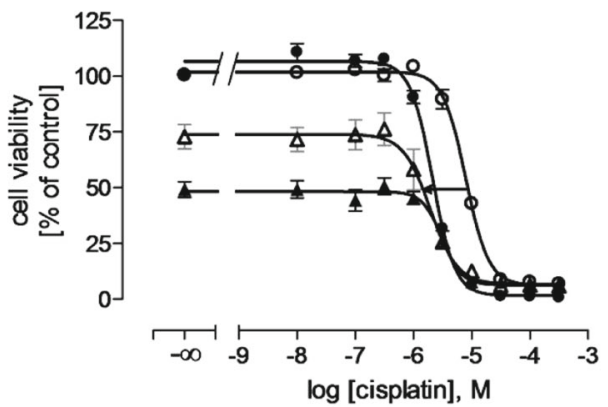

- $\mathrm{HCC} 38$

$\mathrm{IC}_{50} 2.1 \mu \mathrm{M}$

- $\mathrm{HCC} 38+\mathrm{BEZ235}$

- $\mathrm{HCC} 38 \mathrm{CisR}$

$\mathrm{IC}_{50} 3.1 \mu \mathrm{M}$

$\mathrm{IC}_{50} 7.9 \mu \mathrm{M}$

$\Delta \mathrm{HCC} 38 \mathrm{CisR}+\mathrm{BEZ235} \quad \mathrm{IC}_{50} 2.0 \mu \mathrm{M}$

C

cisplatin

BEZ235

PARP (113 kDa)

cleaved PARP (24 kDa)

B-Actin (42 kDa)

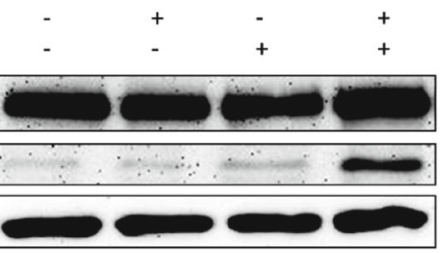

e

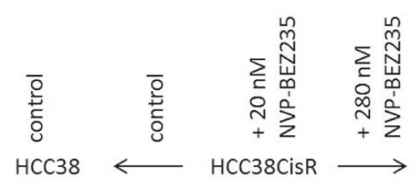

p-EGFR (180 kDa)

p-IGF1R (130 kDa)

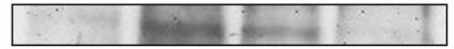

p-Akt (62 kDa)

$\beta$-Actin (42 kDa)

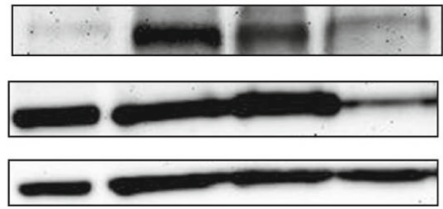

b

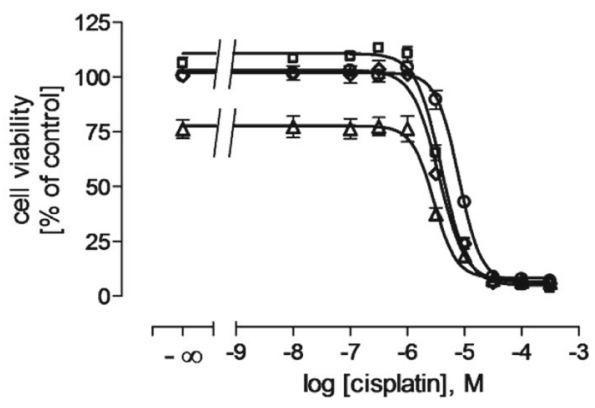

- HCC38CisR $\mathrm{IC}_{50} 7.9 \mu \mathrm{M}$

- HCC38CisR + LY294002 IC $40.0 \mu \mathrm{M}$

○ HCC38CisR + KU0063794 IC $5.6 \mu \mathrm{M}$

$\Delta$ HCC38CisR + KU0063794 + LY294002 IC ${ }_{50} 2.9 \mu \mathrm{M}$

d

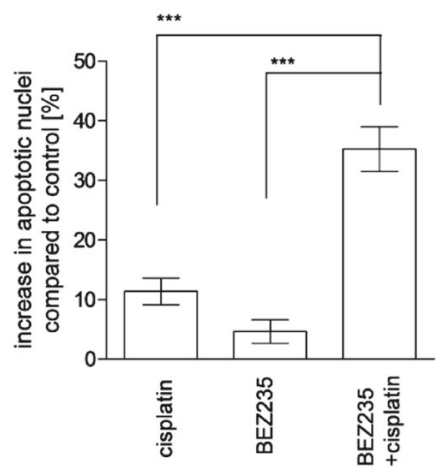

g

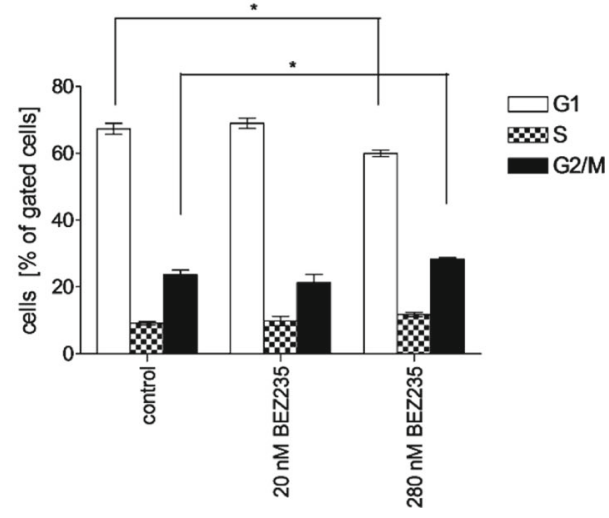

f
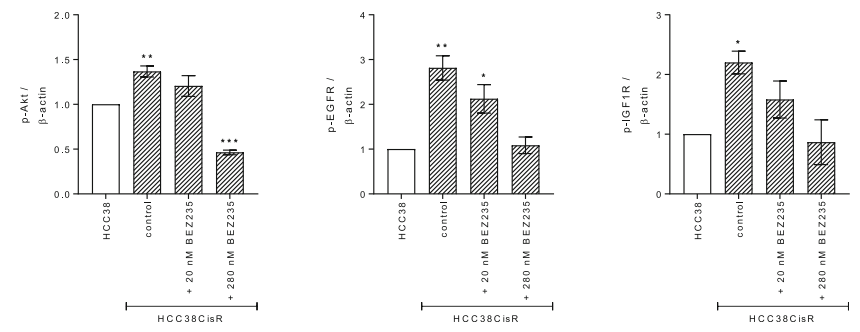

Fig. 3 (See legend on next page.) 


\begin{abstract}
(See figure on previous page.)
Fig. 3 NVP-BEZ235 treatment fully restores cisplatin sensitivity in HCC38CisR. a 20 nM NVP-BEZ235 added 48 h prior to cisplatin treatment significantly reduced $\mathrm{IC}_{50}$ of cisplatin in HCC38CisR $(p<0.001)$ but not in HCC38. b $1 \mu \mathrm{M}$ KU0063794 or $5 \mu \mathrm{M}$ LY294002 or their combination significantly reduced II 50 of cisplatin in HCC38CisR $(p<0.001)$. c Western blot analysis of PARP and cleaved PARP in HCC38CisR used as an indicator of active Caspase 3. For combination of NVP-BEZ235 and cisplatin, $20 \mathrm{nM}$ NVP-BEZ235 was incubated $48 \mathrm{~h}$ prior to addition of $3 \mu \mathrm{M}$ cisplatin for $6 \mathrm{~h}$. $\mathbf{d}$ Induction of apoptosis by NVP-BEZ235 and cisplatin. 20 nM NVP-BEZ235 was incubated 24 h prior to addition of $5 \mu$ M cisplatin for $6 \mathrm{~h}$ followed by $24 \mathrm{~h}$ of recovery. Combination of NVP-BEZ235 with cisplatin increased apoptotic nuclei (35.3 $\pm 3.7 \%)$ compared to cisplatin alone $(11.4 \pm 2.3 \%)$ and NVP-BEZ235 alone (4.6 $\pm 2.0 \%)\left({ }^{* * *} p<0.001\right)$. e Western blot analysis of p-EGFR, p-IGF1R, and p-Akt in HCC38CisR upon $48 \mathrm{~h}$ treatment with $20 \mathrm{nM}$ or $280 \mathrm{nM}$ NVP-BEZ235. $\mathbf{f}$ Densitometric analysis of the protein bands of p-EGFR, p-IGF1R, and p-Akt in HCC38 and HCC38CisR were performed using ImageJ software $(\mathrm{NIH})$. Data are means $\pm \mathrm{SD}, n=3$. All values have been normalized to HCC38 control. Statistical analysis was performed using one-way ANOVA test ${ }^{*} p<0.05,{ }^{* *} p<0.01$, and $\left.{ }^{* * *} p<0.001\right)$. g Effect of 20 nM or 280 nM NVP-BEZ235 on cell cycle in HCC38CisR. 280 nM NVP-BEZ235 gave a slight but significant $\left({ }^{*} p<0.5\right)$ reduction of cells in $G_{1}$ phase $(67.3 \pm 1.6 \%$ vs. 60.0 $\pm 0.9 \%$ in control) accompanied by an increase in cells in $\mathrm{G}_{2} / \mathrm{M}$ phase ( $23.6 \pm 1.4 \%$ vs. $28.3 \pm 0.5 \%$ in control). All data shown are mean $+/-\mathrm{SEM}, \mathrm{n}=3$, except (C/E) showing a representative experiment out of 3
\end{abstract}

$61 \%$ of the scratch was covered by cells (Fig. 4a/b). Treatment with any of the kinase inhibitors reduced migration, however only the combination of $1.5 \mu \mathrm{M}$ NVPAEW541 and $1 \mu \mathrm{M}$ lapatinib showed a significant inhibition of migration (Fig. 4a/b). To exclude that inhibition of migration was only due to reduced proliferation, three different assays evaluating cell viability were performed using the same conditions as applied in the scratch assay: MTT assay, neutral red assay, cell count by flow cytometry. Treatment with NVP-AEW541 or lapatinib or their combination did not affect proliferation (Fig. 4c). Only NVP-BEZ235 significantly reduced cell proliferation compared to untreated control (MTT: $82 \%$, neutral red: $87 \%$, cell count: $81 \%)$. However, NVPBEZ235 did not significantly inhibit migration.

Lastly, we extended the study of NVP-AEW541, lapatinib, NVP-BEZ235 in HCC38 and HCC38CisR to the TNBC cell line MDA-MB231. Similarly to the generation of cisplatin-resistant HCC38CisR, we have generated a 2.1-fold more resistant sub-line named MDA-MB231CisR (Additional file 7A, Table 3). Similar to HCC38CisR, MDA-MB231CisR displayed activated EGFR and IGR1R (Additional file 7B). We then tested combinations of dual and triple combinations of kinase inhibitors and cisplatin by MTT assay (Table 3). In accordance with the results obtained in HCC38 and HCC38CisR (Fig. 3a), NVP-BEZ235 had no effect on cisplatin potency in MDA-MB231 but reversed the 2.1-fold cisplatin resistance of MDAMB231CisR (Table 3). Furthermore, NVP-BEZ235 increased apoptosis induction in combination with cisplatin compared to either compound alone (Additional file 7C). Whereas the combination of NVP-AEW541 plus lapatinib only partially reversed cisplatin resistance in HCC38CisR (Fig. 2g), this combination not only reversed the 2.1-fold resistance of MDA-MB231CisR but shifted cisplatin potency by a factor of 4.8 beyond the sensitivity of MDA-MB231 (Table 3). Notably, similar to the results in HCC38CisR (Fig. 2c), the combination of NVP-
AEW541 and lapatinib showed a highly hyper-additive effect in the induction of apoptosis in both MDAMB231 and MDA-MB231CisR (Additional file 7D).

\section{Discussion}

Among breast cancer, TNBC has a poor prognosis due to the lack of targeted hormone or HER2directed therapy and resistance development against classical cytostatics including cisplatin currently under clinical investigation for TNBC [5]. We have established a cellular model of cisplatin resistance in the TNBC cell line HCC38 to study resistance mechanisms and identify targets for overcoming resistance. The cisplatin resistant cell line labeled HCC38CisR exhibited increased activation observed as phosphorylation of EGFR and IGF1R (Fig. 1). Increased RTK phosphorylation in HCC38CisR was accompanied by faster proliferation (Fig. 1d) and higher susceptibility to EGFR and IGF1R inhibition (Fig. 2a, b) compared to the parental cell line HCC38. By immunostaining, we could demonstrate that an increase in RTK phosphorylation also occurred in HCC38 after short-term $(6 \mathrm{~h})$ cisplatin exposure. However, in contrast to HCC38CisR showing a stable cisplatin resistance with permanent EGFR and IGF1R activation, the shortterm cisplatin-induced receptor phosphorylation in HCC38 nearly vanished after 1 week of recovery (Fig. 1c). Crosstalk between RTKs as well as the ability of cancer cells to switch between different growth factor receptor pathways is well described [9]. Therefore, lapatinib and NVP-AEW541 were selected to inhibit both activated RTKs in HCC38CisR simultaneously. According to the Cancer Cell Line Encyclopedia [22], EGFR mutations possibly impairing the effect of lapatinib are not described for HCC38. Activation of EGFR and IGF1R was observed in HCC38CisR, but RTK activation is rather associated with than a cause of cisplatin resistance in HCC38CisR as we could not restore cisplatin sensitivity by inhibition of these RTKs with lapatinib and NVP-AEW541 (Fig. 2g) even 


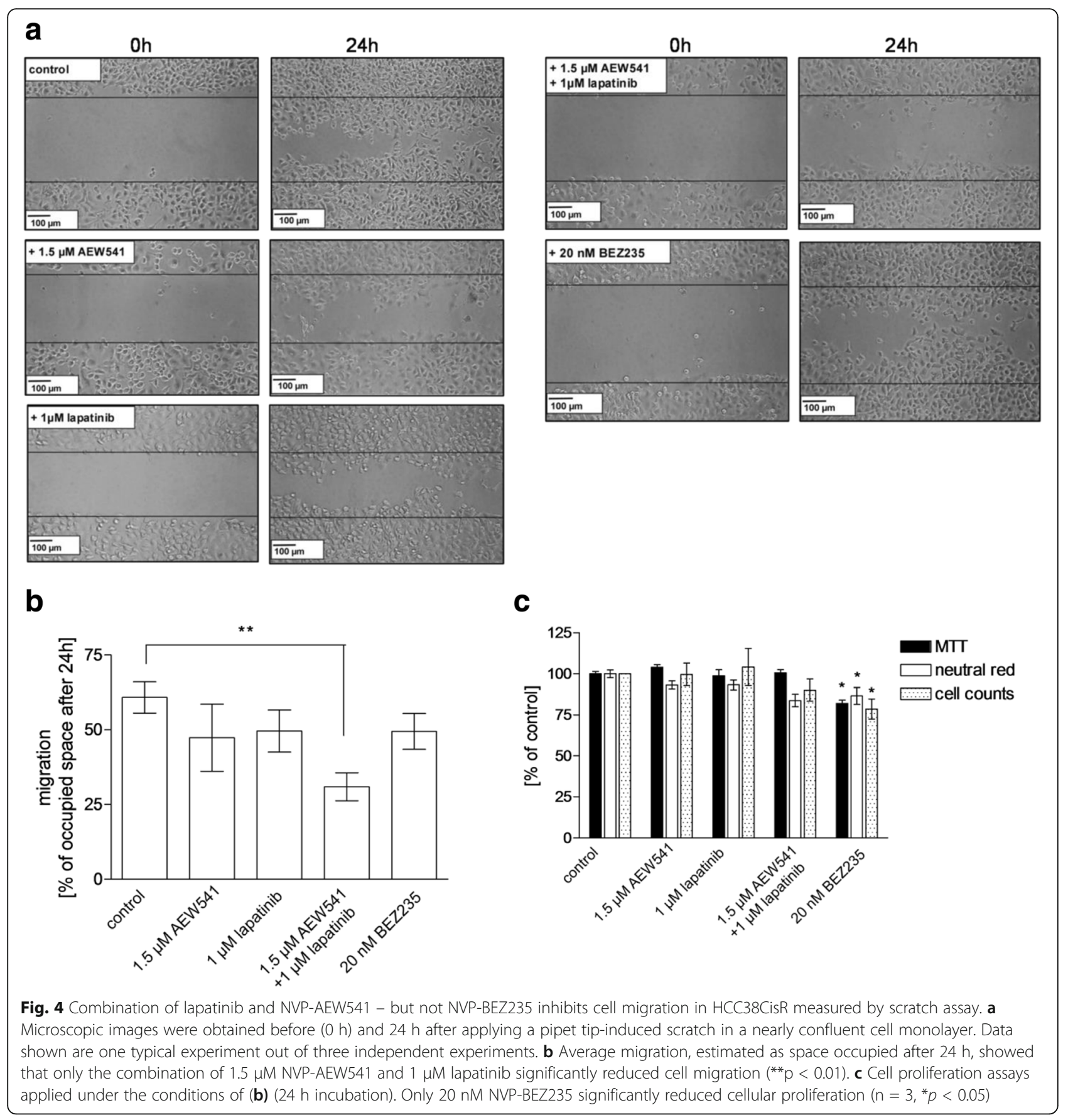

though both compounds were shown to successfully inhibit EGFR and IGF1R phosphorylation (Fig. 2e, f). Notably, in the highly cisplatin-resistant cell line MDA-MB231CisR ( $\mathrm{IC}_{50} 44.0 \mu \mathrm{M}$ ), we found a 4.8 -fold resensitization for cisplatin upon pretreatment with NVP-AEW541 and lapatinib ( $\mathrm{IC}_{50} 9.16 \mu \mathrm{M}$, Table 3). We could demonstrate synergy of lapatinib and NVPAEW541 with respect to inhibition of cell viability (Table 1) and apoptosis induction (Fig. 2c, Additional file 7D). Coincubation with NVP-AEW541 reduced $\mathrm{IC}_{50}$ of lapatinib nearly 7 -fold (Fig. 1a). This effect may be of clinical importance as the resulting $\mathrm{IC}_{50}$ of $0.88 \mu \mathrm{M}$ is lower than the reported $\mathrm{c}_{\max }$ of lapatinib $(1.7-4 \mu \mathrm{M})[23,24]$. In cell cycle analysis we could show that the combination of EGFR and IGF1R inhibition resulted in an increase in cells in $G_{1}$ phase. This might be one possible mechanism leading to reduced cell proliferation. These results are in accordance with studies performed on adrenocortical carcinomas applying EGFR and IGF1R inhibitors [25]. 
Table $3 I_{50}$ values $(\mu M)$ from MTT assays and corresponding shift factors (SF) of cisplatin alone and after $48 \mathrm{~h}$ pretreatment with $1.5 \mu \mathrm{M}$ NVP-AEW541, 2 MM lapatinib, 20 nM NVP-BEZ235, or $1.5 \mu \mathrm{M}$ NVP-AEW541 plus $2 \mu \mathrm{M}$ lapatinib, respectively, in MDA-MB231 and MDA-MB231CisR cells

\begin{tabular}{|c|c|c|c|c|}
\hline \multirow[t]{2}{*}{ Compound } & \multicolumn{2}{|c|}{ MDA-MB231 } & \multicolumn{2}{|c|}{ MDA-MB231 CisR } \\
\hline & $\mathrm{IC}_{50}$ & SF & $\mathrm{IC}_{50}$ & SF \\
\hline cisplatin & 20.9 & - & 44.0 & - \\
\hline cisplatin + AEW541 & 22.8 & 0.9 & 23.3 & 1.9 \\
\hline cisplatin + lapatinib & 24.1 & 0.9 & 24.7 & 1.8 \\
\hline cisplatin + BEZ235 & 22.8 & 0.9 & 21.8 & $2.0^{*}$ \\
\hline cisplatin + AEW541 + lapatinib & 10.1 & $2.1^{*}$ & 9.16 & $4.8^{*}$ \\
\hline
\end{tabular}

Another effect of the combination of lapatinib and NVP-AEW541 in HCC38CisR is the reduction of cell migration (Fig. 4a/b) which was not due to decreased proliferation as shown by simultaneously performed proliferation assays (Fig. 4c). Migration of cancer cells serves as a marker for invasion and the potential to form metastases. As TNBC has a high risk for metastases [26], drugs reducing migration may be valuable in treating TNBC. Although the advantages of combining RTK inhibitors have been shown several years ago [27], the in vitro results have not yet been transferred into clinical benefits [14]. Taking into account that the approach of combining NVP-AEW541 and lapatinib showed only synergy in HCC38CisR but not in HCC38, it might be of value to select tumors according to their RTK activation. Our study demonstrates that the phosphorylation status of RTKs predicts response to the combination of lapatinib and NVP-AEW541 (in HCC38CisR and MDA-MB231CisR) whereas receptor expression showed only marginal differences between non-responding $\mathrm{HCC} 38$ and responding HCC38CisR. Therefore, the selection of targeted therapies by receptor phosphorylation rather than receptor expression might be an approach for further studies.

Lapatinib and NVP-AEW541 were ineffective to restore cisplatin sensitivity in HCC38CisR (Fig. 2g). However, Akt was stronger phosphorylated in HCC38CisR than in untreated or short-term $(6 \mathrm{~h})$ cisplatin-treated HCC38 (Fig. 1c), assuming an increased activation in the course of cisplatin resistance development. Lapatinib and NVPAEW541 did not influence downstream Akt phosphorylation (Fig. 2e) suggesting further mechanisms conserving Akt activation [28]. It has been shown that dual inhibition of two kinases in IGF1R signaling pathway is superior to applying only single agents in the TNBC cell line MDAMB-231 [29]. Therefore, we chose the dual PI3K/mTOR inhibitor NVP-BEZ235 to address increased Akt activation in HCC38CisR. Synergy of NVP-BEZ235 has already been demonstrated for paclitaxel in colon cancer cells [30] and carboplatin in a triple negative breast cancer cell line [31]. Additionally, NVP-BEZ235 has already proven its ability to enhance cisplatin sensitivity in cisplatin resistant bladder cancer cell lines [32].

In our study, NVP-BEZ235 could fully restore cisplatin sensitivity in the cisplatin-resistant TNBC cell line HCC38CisR and acted synergistically with cisplatin (Fig. 3a/d, Table 2). Using KU0063794 and LY2940002, we could demonstrate that it was not sufficient to inhibit mTOR or PI3K alone, respectively, to obtain the NVP-BEZ235-induced effect on cisplatin sensitivity (Fig. 3b). Combining KU0063794 and LY294002 and thereby mimicking the dual inhibition of NVP-BEZ235 increased the effect of each compound alone on cisplatin sensitivity (Fig. 3b). Nevertheless, NVP-BEZ235 was slightly more effective than the combination of KU0063794 and LY294002. Other studies have shown that mTOR inhibition might result in only transient decrease or even increase of phospho-Akt (p-Akt) caused by feedback activation [31, 33]. Thus, these and our results allow the conclusion that the combination of PI3K and mTOR inhibition is preferred over mTOR inhibition alone for cisplatin sensitization. Lastly, synergy between NVP-BEZ235 and cisplatin was not observed in HCC38 even though Akt showed some activation, however lower than in HCC38CisR. This indicates that NVP-BEZ235 enhances cisplatin sensitivity if - next to Akt activation - upstream RTKs such as EGFR and IGF1R are activated. Activated RTKs plus activated Akt may thus serve as potential biomarkers for the use of NVP-BEZ235 in combination with cisplatin in TNBC. These results in HCC38CisR were corroborated by data obtained with MDA-MB231CisR (Table 3, Additional file 7).

\section{Conclusions}

Taken together, activation of EGFR and IGF1R and their downstream signaling pathway kinase Akt is associated with resistance induced by long-term treatment with cisplatin in the TNBC cell line HCC38 and in MDA-MB231. Based on these results, two approaches for treating cisplatin resistant cell lines are presented: 1) Simultaneous inhibition of EGFR and IGF1R by lapatinib and NVP-AEW541 is highly synergistic and results in the induction of apoptosis. Furthermore, co-treatment with lapatinib and NVPAEW541 may increase cisplatin sensitivity as seen in MDA-MB231CisR. 2) Co-treatment of cisplatinresistant TNBC cell lines with the $\mathrm{PI} 3 \mathrm{~K} / \mathrm{mTOR}$ inhibitor NVP-BEZ235 and cisplatin is synergistic, fully reversed acquired cisplatin resistance, and may thus constitute a targeted treatment option for cisplatinresistant TNBC. 


\section{Additional files}

Additional file 1: plC50 values and standard error of the mean. plC50 values, errors, and IC50 values of all MTT assays performed in this study are listed. (DOC $70 \mathrm{~kb}$ )

Additional file 2: cell cycle distribution. The cell cycle distribution in HCC38 after treatment with NVP-AEW541, lapatinib or both compounds is displayed as bar graph. (DOCX $30 \mathrm{~kb}$ )

Additional file 3: MTT assay of combination of RTK inhibitors with cisplatin. Influence of $48 \mathrm{~h}$ preincubation with $1.5 \mu \mathrm{M}$ NVP-AEW541, $1 \mu \mathrm{M}$ lapatinib or a combination of both compounds on cisplatin sensitivity in HCC38. (DOCX $173 \mathrm{~kb}$ )

Additional file 4: MTT assay of NVP-BEZ235. Effect of NVP-BEZ235 on cell viability determined by MTT assay. (DOCX 29 kb)

Additional file 5: Western blot of cleaved PARP upon NVP-BEZ235 and cisplatin treatment. Western Blot on cleaved PARP after treatment of HCC38 with 20 nM NVP-BEZ235 or $2 \mu \mathrm{M}$ cisplatin or a combination of both compounds. (DOCX $80 \mathrm{~kb}$ )

Additional file 6: induction of apoptosis in HCC38 upon NVP-BEZ235 and cisplatin treatment. Induction of apoptotic nuclei in HCC38 after treatment with $2 \mu \mathrm{M}$ cisplatin, $20 \mathrm{nM}$ NVP-BEZ235 or a combination of both compounds. (DOCX $25 \mathrm{~kb}$ )

Additional file 7: Characterization of MDA-MB231 and cisplatin-resistant MDA-MB231CisR. MDA-MB231 and cisplatin-resistant MDA-MB231CisR cells were characterized by MTT assay, phospho-RTK status, and induction of apoptosis upon kinase inhibitor and cisplatin treatment. (DOCX $141 \mathrm{~kb}$ )

\section{Abbreviations}

Akt: Protein kinase B; Cl: Combination index; $\mathrm{C}_{\max }$ : Maximum serum concentration; EGFR: Human epidermal growth factor receptor; ER: Estrogen receptor; ErbB2: Human epidermal growth factor receptor 2; ErbB3: Human epidermal growth factor receptor 3; ErbB4: Human epidermal growth factor receptor 4; HRP: Horseradish peroxidase; $\mathrm{C}_{50}$ : Half-maximum inhibitory concentration; IGF1: Insulin-like growth factor 1; IGF1R: Insulin-like growth factor 1 receptor; mTOR: mechanistic target of rapamycin; MTT: 3-(4,5Dimethylthiazol-2-yl)-2,5-diphenyltetrazolium bromide; p-Akt: phospho-Akt; PARP: Poly ADP ribose polymerase; p-EGFR: phospho-EGFR; p-ErbB2: phospho-ErbB2; p-ErbB3: phospho-ErbB3; p-ErbB4: phospho-ErbB4; PI3K: Phosphatidylinositol-3-kinase; p-IGF1R: phospho-IGF1R; PR: Progesterone receptor; RTK: Receptor tyrosine kinase; TNBC: Triple negative breast cancer

\section{Acknowledgements}

Not applicable.

\section{Funding}

This work was funded by grants from the Bundesministerium für Wirtschaft (BMWi) AiF/ZIM project KF2388801UL9 to MUK. The funding body did not interfere nor had any objections regarding the design of the study and collection, analysis, and interpretation of data and in writing the manuscript.

\section{Availability of data and materials}

All data generated or analysed during this study are included in this published article and its supplementary information files. In addition, raw datasets used and/or analysed during the current study are available from the corresponding author on reasonable request

\section{Authors' contributions}

KG conceived the study, designed the experiments, collected and analyzed the data, interpreted the results and wrote the manuscript. $\mathrm{AH}$ made substantial contributions to the conception of the study, performed experiments, analyzed data, and was substantially involved in writing and revising the manuscript. LHE was involved in data acquisition and data analysis. MUK conceived the study, designed the experiments, analyzed the data, interpreted the results, wrote the manuscript and revision of the manuscript, and provided financial support. All authors read and approved the final manuscript and agreed to be accountable for all aspects of the work.
Ethics approval and consent to participate

Not applicable.

\section{Consent for publication}

Not applicable.

\section{Competing interests}

The authors declare that they have no competing interests.

\section{Publisher's Note}

Springer Nature remains neutral with regard to jurisdictional claims in published maps and institutional affiliations.

Received: 20 September 2016 Accepted: 19 October 2017

Published online: 03 November 2017

\section{References}

1. Global Burden of Disease Cancer C, Fitzmaurice C, Dicker D, Pain A, Hamavid H, Moradi-Lakeh M, MF MI, Allen C, Hansen G, Woodbrook R, et al. The global burden of cancer 2013. JAMA Oncol. 2015;1(4):505-27.

2. Papa A, Caruso D, Tomao S, Rossi L, Zaccarelli E, Tomao F. Triple-negative breast cancer: investigating potential molecular therapeutic target. Expert Opin Ther Targets. 2015:19(1):55-75.

3. Foulkes WD, Smith IE, Reis JS. Triple-negative breast cancer. New Engl J Med. 2010:363(20):1938-48.

4. von Minckwitz G, Schneeweiss A, Loibl S, Salat C, Denkert C, Rezai M, Blohmer JU, Jackisch C, Paepke S, Gerber B et al: Neoadjuvant carboplatin in patients with triple-negative and HER2-positive early breast cancer (GeparSixto; GBG 66): a randomised phase 2 trial. Lancet Oncol 2014, 15(7):747-756.

5. Isakoff SJ, Mayer EL, He L, Traina TA, Carey LA, Krag KJ, Rugo HS, Liu MC, Stearns V, Come SE, et al. TBCRC009: a multicenter phase II clinical trial of platinum Monotherapy with biomarker assessment in metastatic triplenegative breast cancer. J Clin Oncol. 2015:33(17):1902-9.

6. Galluzzi L, Senovilla L, Vitale I, Michels J, Martins I, Kepp O, Castedo M, Kroemer G. Molecular mechanisms of cisplatin resistance. Oncogene. 2012; 31(15):1869-83.

7. Engelke LH, Hamacher A, Proksch P, Kassack MU. Ellagic acid and Resveratrol prevent the development of Cisplatin resistance in the epithelial ovarian cancer cell line A2780. J Cancer. 2016;7(4):353-63.

8. Eckstein N, Servan K, Hildebrandt B, Politz A, von Jonquieres G, WolfKummeth S, Napierski I, Hamacher A, Kassack MU, Budczies J et al: Hyperactivation of the insulin-like growth factor receptor I signaling pathway is an essential event for cisplatin resistance of ovarian cancer cells. Cancer Res 2009, 69(7):2996-3003.

9. Jin Q, Esteva FJ. Cross-talk between the ErbB/HER family and the type I insulin-like growth factor receptor signaling pathway in breast cancer. J Mammary Gland Biol Neoplasia. 2008;13(4):485-98.

10. Hankinson SE, Willett WC, Colditz GA, Hunter DJ, Michaud DS, Deroo B, Rosner B, Speizer FE, Pollak M. Circulating concentrations of insulin-like growth factor-I and risk of breast cancer. Lancet. 1998;351(9113):1393-6.

11. Law JH, Habibi G, Hu K, Masoudi H, Wang MY, Stratford AL, Park E, Gee JM, Finlay $\mathrm{P}$, Jones $\mathrm{HE}$, et al. Phosphorylated insulin-like growth factor-i/insulin receptor is present in all breast cancer subtypes and is related to poor survival. Cancer Res. 2008;68(24):10238-46.

12. Pollak $M$. The insulin and insulin-like growth factor receptor family in neoplasia: an update. Nat Rev Cancer. 2012;12(3):159-69.

13. Huang F, Greer A, Hurlburt W, Han X, Hafezi R, Wittenberg GM, Reeves K, Chen J, Robinson D, Li A, et al. The mechanisms of differential sensitivity to an insulin-like growth factor-1 receptor inhibitor (BMS-536924) and rationale for combining with EGFR/HER2 inhibitors. Cancer Res. 2009;69(1):161-70.

14. Beckwith H, Yee D. Minireview: were the IGF Signaling inhibitors all bad? Mol Endocrinol. 2015:29(11):1549-57.

15. Gosepath EM, Eckstein N, Hamacher A, Servan K, von Jonquieres G, Lage H, Gyorffy B, Royer HD, Kassack MU: Acquired cisplatin resistance in the head-neck cancer cell line Cal27 is associated with decreased DKK1 expression and can partially be reversed by overexpression of DKK1. Int J Cancer 2008, 123(9):2013-2019.

16. Chou TC. Drug combination studies and their synergy quantification using the Chou-Talalay method. Cancer Res. 2010;70(2):440-6. 
17. Borenfreund $\mathrm{E}$, Shopsis $\mathrm{C}$. Toxicity monitored with a correlated set of cell-culture assays. Xenobiotica; the fate of foreign compounds in biological systems. 1985; 15(8-9):705-11.

18. Schneider CA, Rasband WS, Eliceiri KW. NIH image to ImageJ: 25 years of image analysis. Nat Methods. 2012;9(7):671-5.

19. McDermott M, Eustace AJ, Busschots S, Breen L, Crown J, Clynes M, O'Donovan N, Stordal B. In vitro development of chemotherapy and targeted therapy drug-resistant cancer cell lines: a practical guide with case studies. Front Oncol. 2014;4:40.

20. Jones HE, Gee JM, Hutcheson IR, Knowlden JM, Barrow D, Nicholson Rl. Growth factor receptor interplay and resistance in cancer. Endocr Relat Cancer. 2006;13(Suppl 1):S45-51.

21. Serra V, Markman B, Scaltriti M, Eichhorn PJ, Valero V, Guzman M, Botero ML, Llonch E, Atzori F, Di Cosimo S et al: NVP-BEZ235, a dual PI3K/mTOR inhibitor, prevents PI3K signaling and inhibits the growth of cancer cells with activating PI3K mutations. Cancer Res 2008, 68(19):8022-8030.

22. Barretina J, Caponigro G, Stransky N, Venkatesan K, Margolin AA, Kim S, Wilson CJ, Lehar J, Kryukov GV, Sonkin D, et al. The cancer cell line Encyclopedia enables predictive modelling of anticancer drug sensitivity. Nature. 2012;483(7391):603-7.

23. Burris HA 3rd, Hurwitz HI, Dees EC, Dowlati A, Blackwell KL, O'Neil B, Marcom PK, Ellis MJ, Overmoyer B, Jones SF, et al. Phase I safety, pharmacokinetics, and clinical activity study of lapatinib (GW572016), a reversible dual inhibitor of epidermal growth factor receptor tyrosine kinases, in heavily pretreated patients with metastatic carcinomas. J Clin Oncol. 2005:23(23):5305-13.

24. LoRusso PM, Jones SF, Koch KM, Arya N, Fleming RA, Loftiss J, Pandite L, Gadgeel S, Weber BL, Burris HA 3rd. Phase I and pharmacokinetic study of lapatinib and docetaxel in patients with advanced cancer. J Clin Oncol. 2008;26(18):3051-6.

25. Xu L, Qi Y, Xu Y, Lian J, Wang X, Ning G, Wang W, Zhu Y. Co-inhibition of EGFR and IGF1R synergistically impacts therapeutically on adrenocortical carcinoma. Oncotarget. 2016;

26. Bosch A, Eroles P, Zaragoza R, Vina JR, Lluch A. Triple-negative breast cancer: molecular features, pathogenesis, treatment and current lines of research. Cancer Treat Rev. 2010;36(3):206-15.

27. Camirand A, Zakikhani M, Young F, Pollak M. Inhibition of insulin-like growth factor-1 receptor signaling enhances growth-inhibitory and proapoptotic effects of gefitinib (Iressa) in human breast cancer cells. Breast Cancer Res : BCR. 2005;7(4):R570-9.

28. Altomare DA, Testa JR. Perturbations of the AKT signaling pathway in human cancer. Oncogene. 2005;24(50):7455-64.

29. Ayub A, Yip WK, Seow HF: Dual treatments targeting IGF-1R, PI3K, mTORC or MEK synergize to inhibit cell growth, induce apoptosis, and arrest cell cycle at G1 phase in MDA-MB-231 cell line. Biomed Pharmacother = Biomedecine \& pharmacotherapie 2015, 75:40-50.

30. Zou H, Li L, Garcia Carcedo I, Xu ZP, Monteiro M, Gu W. Synergistic inhibition of colon cancer cell growth with nanoemulsion-loaded paclitaxel and PI3K/mTOR dual inhibitor BEZ235 through apoptosis. Int J Nanomedicine. 2016;11:1947-58.

31. Montero JC, Esparis-Ogando A, Re-Louhau MF, Seoane S, Abad M, Calero R, Ocana A, Pandiella A. Active kinase profiling, genetic and pharmacological data define $m T O R$ as an important common target in triple-negative breast cancer. Oncogene. 2014;33(2):148-56.

32. Moon du G, Lee SE, Oh MM, Lee SC, Jeong SJ, Hong SK, Yoon CY, Byun SS, Park HS, Cheon J: NVP-BEZ235, a dual PI3K/mTOR inhibitor synergistically potentiates the antitumor effects of cisplatin in bladder cancer cells. Int J Oncol 2014, 45(3):1027-1035.

33. Randodrik-Outmezguine VS, Chandarlapaty S, Pagano NC, Poulikakos PI, Scaltriti M, Moskatel E, Baselga J, Guichard S, Rosen N. mTOR kinase inhibition causes feedback-dependent biphasic regulation of AKT signaling. Cancer Discov. 2011;1(3):248-59.

\section{Submit your next manuscript to BioMed Central and we will help you at every step:}

- We accept pre-submission inquiries

- Our selector tool helps you to find the most relevant journal

- We provide round the clock customer support

- Convenient online submission

- Thorough peer review

- Inclusion in PubMed and all major indexing services

- Maximum visibility for your research

Submit your manuscript at www.biomedcentral.com/submit
Biomed Central 